\title{
Lifetime Maximization with Inter-Session Network Coding in Energy Constrained Wireless Networks
}

\author{
Lianghui Ding ${ }^{1}$, Ping $\mathrm{Wu}^{1}$, Hao Wang ${ }^{2,1}$, Zhiwen $\mathrm{Pan}^{2}$, Xiaohu You ${ }^{2}$ \\ ${ }^{1}$ Signals and Systems, Dept. of Engineering Sciences, Uppsala University, Uppsala, Sweden \\ ${ }^{2}$ National Mobile Communication Research Laboratory, Southeast University, Nanjing, China \\ \{lhding, ping.wu\}@angstrom.uu.se, \{hao_wang, pzw, xhyu\}@seu.edu.cn
}

\begin{abstract}
This paper deals with lifetime maximization for energy constrained wireless networks with inter-session network coding. The problem of lifetime maximization is first formulated, and then transformed into a linear programming problem. Using Lagrangian dual decomposition, it is further converted into a dual problem that consists of two subproblems: lifetime optimization and network optimization, and then it is solved by utilizing dual subgradient method. Convex combination is used for primary recovery from the dual solutions. Performance of the algorithm is evaluated through simulation. Results illustrate that theoretical analysis matches practical calculation well, and that inter-session network coding can prolong the network lifetime up to $50 \%$ compared with the one without network coding.
\end{abstract}

Keywords-network coding, lifetime maximization, wireless networks, linear programming, subgradient method

\section{INTRODUCTION}

The lifetime of a energy constrained network (e.g, wireless sensor network) is often limited by the available energy of network nodes, thus lifetime maximization has become an interesting research topic and been investigated extensively in recent years [1], [2]. However, little research has been done on lifetime maximization with network coding.

The concept of network coding was firstly constructed by Ahlswede in [3], which proved that the achievable rate of multicast is the max-flow from the source node to all sink nodes, and can be realized with block coding. After that, many research has been done on various aspects of network coding, such as coding approaches [4], [5], practical application of network coding [6]-[9], etc.

The basic idea of network coding is to equip routers with processing ability, and compress native packets utilizing spatial redundancy among nodes to reduce the occupied network bandwidth. Since wireless signal is inherently broadcast, there is sufficient spatial redundancy that can be used by network coding, much research has been done to improve the network throughput with network coding in wireless networks [7], [10].

Heuristically, the advantage of network coding is to decrease the number of transmissions in wireless networks, hence the power of some bottleneck nodes can be saved and the lifetime of the whole network is prolonged. Performance of lifetime

This work is supported mainly by VINNOVA Sweden (Grant 2008- 00954); and in part by CSC China, the Ministry of Science and Technology China through International Science and Technology Cooperation Program under grant 2008DFA12090 and National Communications Research Laboratory Program (2009A02). extension with network coding in some specific scenarios is presented in [11]. However, application of network coding to maximize lifetime of an energy constrained network is not so straightforward, since the performance of network coding depends on how the routing and scheduling are designed [5], [6], [12]. Until now most of the works are focused on heuristically designed network coding schemes that are suitable for energy constrained networks [13], while theoretical analysis of lifetime maximization with network coding has not received much attention.

Network coding can be performed in two categories: intersession and intra-session. In inter-session network coding the packets from different commodities are coded into one packet, while in intra-session network coding only packets from the same commodity can be coded. Optimal intra-session network coding can be analyzed with optimization tools easily [6], while only specific optimal inter-session network coding can be analyzed such as in [8], [14], and general optimal intersession network coding is still an open question.

In this paper, we investigate the lifetime maximization for energy constrained wireless networks with inter-session network coding. Flows with the same destination nodes are formulated as a commodity, and network coding is allowed in the newly formulated commodity. We formulate the problem of lifetime maximization with inter-session network coding as a linear programming problem, transform it into a convex optimization problem, and then solve it with subgradient method in a distributed manner. The dual problem is decomposed into two subproblems. One is lifetime maximization with total energy constraint at each node, and the other is routing and scheduling under the flow conservation and physical rates constraints. Through mathematical analysis, we simplify the latter subproblem into a maximum weighted hypergraph matching problem, which is solved approximately with only local information exchange [10]. After that we give the distributed algorithm and evaluate it through simulation. The results illustrate that the theoretical analysis matches the practical calculation well, and network coding can effectively prolong the network lifetime.

The rest of the paper is arranged as follows. In Section II, network model, interference model and network coding are presented, and the definition of network lifetime is given. Then, we formulate the problem, present the solution with subgradient method, dual decomposition, and primary recov- 
ery, and the distributed algorithm in Section III. After that, the performance of the algorithm is evaluated and compared with the one without network coding in Section IV. Finally the paper is concluded in Section V.

\section{Preliminaries}

\section{A. Network Model}

A static wireless network is modeled as a directed hypergraph $\mathcal{G}=\{\mathcal{N}, \mathcal{L}\}$, where $\mathcal{N}$ is the set of nodes, $\mathcal{L}$ is the set of hyperarcs. A hyperarc $\{i, J\} \in \mathcal{L}$ denotes a multicast connection between node $i$ and a subset of its neighboring nodes, which means that the transmission of node $i$ can be correctly received by all the nodes in $J$ with probability 1 . Let $N(i)$ denote the set of neighboring nodes of node $i$. Note that $J \subseteq N(i)$, but does not always equal to $N(i)$.

A unicast flow is defined as the data transfer from a source node to a destination node, while a multicast flow is that from the source node to all its destination nodes. Multicast flows with the same destination nodes constitute a commodity. Throughout the paper, we use $c$ and $C$ to denote a specific commodity and the set of all commodities, respectively; $\mathcal{S}^{c}$ and $\mathcal{D}^{c}$ to denote the set of source nodes and the set of destination nodes of commodity $c$, respectively; and $s^{c}$ and $d^{c}$ to denote a source node and a destination node of commodity $c$, respectively.

Primary interference model and K-hop interference model are two widely accepted resource sharing models. With a specific resource sharing model, the achievable rate region of the network depends on the scheduling policy. Let $\pi$, $\Pi$ denote a scenario of scheduling and the set of all scheduling scenarios, the achievable rate region of the network with timesharing will be

$$
\operatorname{Co}(\Pi)=\left\{r_{i J}: \sum_{\pi \in \Pi} \alpha_{\pi} r_{i J}^{\pi}, \alpha_{\pi} \geq 0, \sum_{\pi \in \Pi} \alpha_{\pi}=1\right\}
$$

where $\alpha_{\pi}$ is the time shared by scheduling $\pi, r_{i J}^{\pi}$ is the rate of hyperarc $(i, J)$ in $\pi, C o(r)$ is the convex hull of all achievable rates set under the scheduling policy set $\Pi$.

\section{B. Network Coding}

As explained in Section I, inter-session network coding in the constituted commodity is considered in this paper. Furthermore, we use one-hop network coding, in which the coded packets have to be decoded by immediate one-hop receivers. Linear XOR [7] is utilized as the packet operation, in which network coding is similar to $c=a \oplus b$. Here, $a$ and $b$ are input packets, and $c$ is the output packet after XOR. To simplify XOR operation, the lengths of all packets are assumed to be equal.

\section{Network Lifetime}

The lifetime is defined as the time period during which all nodes functions properly, and the formal definition is defined as follows.

Let $E_{i}, e_{i J}^{t}$, and $e_{j I}^{r}$ denote the total energy of node $i$, power cost of transmitting an data unit from node $i$ to $J$ and receiving an data unit from $j$ by $i$, respectively, where $(i, J) \in \mathcal{L}$, $(j, I) \in \mathcal{L}, i \in I$. Let $f_{i J}^{c}$ denote the physical transmission rate of commodity $c$ from node $i$ to $J$, the lifetime of node $i$ can be expressed as

$$
T_{i}=\frac{E_{i}}{\sum_{c \in \mathcal{C}}\left[\sum_{(i, J) \in \mathcal{L}} e_{i J}^{t} f_{i J}^{c}+\sum_{(j, I) \in \mathcal{L}, i \in I} e_{j I}^{r} f_{j I}^{c}\right]}
$$

which is determined by the total energy $E_{i}$ of node $i$ and the data transmitting and receiving rates.

Then the lifetime of the network is given by

$$
T=\min _{i \in \mathcal{N}} T_{i}
$$

This definition tells that the network will die when the energy of one node is exhausted. Note that by using lifetime maximized routing, this definition is equivalent to the time that the network is disconnected and at least one demand can not be served by the network anymore.

\section{Problem Formulation and Solution}

\section{A. Problem Formulation}

According to the definitions and assumptions discussed in Section II, the lifetime maximization with inter-session network coding can be formulated as follows

$$
\begin{gathered}
\text { s.t. } \sum_{(i, J) \in \mathcal{L}} \sum_{j \in J} g_{i J j}^{c s d}-\sum_{j \in \mathcal{N}} \sum_{(j, I) \in \mathcal{L}, i \in I} g_{j I i}^{c s d}=\sigma_{i}^{c s}, \quad \forall i, c, s, d \\
\sum_{s \in \mathcal{S}^{c}} \sum_{j \in J} g_{i J j}^{c s d} \leq f_{i J}^{c}, \quad \forall\{i, J\}, c, d \\
\sum_{c \in C} f_{i J}^{c} \leq r_{i J}, \quad \forall\{i, J\} \\
\left(\sum_{i \in C} \sum_{(i, J) \in \mathcal{L}} e_{i}^{t} f_{i J}^{c}+\sum_{c \in C} \sum_{j \in \mathcal{N}} \sum_{(j, I) \in \mathcal{L}, i \in I} e_{i}^{r} f_{j I}^{c}\right) T \leq E_{i}, \quad \forall i
\end{gathered}
$$

In Equation (5), $g_{i J j}^{c s d}$ denotes the effective transmission rate from node $i$ to node $j \in J$ for the flow in commodity $c$, which is from the source node $s$ to the destination node $d$. The rate $\sigma_{i}^{c s}$ is defined as

$$
\sigma_{i}^{c s}=\left\{\begin{array}{cl}
x_{s}^{c}, & \text { if } i \in \mathcal{S}^{c}, \\
-x_{s}^{c}, & \text { if } i \in \mathcal{D}^{c}, \\
0, & \text { otherwise. }
\end{array}\right.
$$

where $x_{s}^{c}$ is the output rate from the source node $s$ in commodity $c$.

Equation (5) describes the flow conservation law: at each node the total output rate of the flow in commodity $c$ from source node $s$ to destination node $d$ should be equal to the corresponding input rate.

Equation (6) explains that the sum of rates transmitted on the hyperarc $\{i, J\}$ to destination $d$ in commodity $c$ is smaller than the physical rate $f_{i J}^{c}$ allocated to commodity $c$. For each node $j \in J$, it can only receive the data from 
one source in one transmission from node $i$, while the data can be forwarded to different destination nodes. With this constraint, both broadcast and network coding are allowed. Without network coding, the data from one source node is broadcasted to all nodes in $J$. With network coding, one transmission may serve several flows from different sources to different destinations simultaneously. Note that broadcast is also allowed when using network coding.

The constraints on physical rates are given in Equations (7) and (8). The total transmission rate of $(i, J)$ should be less than the available physical rate $r_{i J}$, while the set of $r_{i J}$ is limited by achievable rate region.

Equation (9) presents the energy constraint on each node. The product of energy cost rate and lifetime should be less than the total energy of each node.

To obtain an equivalent linear programing problem and distribute the lifetime constraint to each node, we use $\sum_{i \in \mathcal{N}} q_{i}^{2}$ in place of $q=1 / T$ and supplement constraints on equality among lifetime of neighboring nodes [2]. The new problem is given in te following manner

$$
\begin{gathered}
(\mathrm{P} 3) \quad \min \sum_{i \in \mathcal{N}} q_{i}^{2} \\
\text { s.t. Equations }(5)-(8) \\
q_{i}=q_{j}, \quad \forall i, \quad \forall j \in N(i) \\
q_{i}>0, \quad \forall i \\
\sum_{c \in C} \sum_{(i, J) \in \mathcal{L}} e_{i}^{t} f_{i J}^{c}+\sum_{c \in \mathcal{C}} \sum_{j \in \mathcal{N}} \sum_{(j, I)} e_{i n \mathcal{L}, i \in I}^{r} f_{j I}^{c} \leq q E_{i}, \quad \forall i
\end{gathered}
$$

\section{B. Lagrangian Dual}

Let $\lambda_{i}^{c s d}, \rho_{i} \geq 0$, and $\mu_{i j}$ denote the Lagrangian multipliers for equations (5), (14), and (12) respectively, we will obtain the partial Lagrangian dual $L(q, g ; \lambda, \rho, \mu)$, and the dual objective function is

$$
W(\lambda, \rho, \mu)=\inf _{q, g} L(q, g ; \lambda, \rho, \mu)
$$

We can assume that there always exist flow distribution with network coding for all flow demands, which satisfies the flow conservation law and strictly satisfies the rate constraints in the primal problem. We can also choose a large enough $q_{i}$, $\forall i$ to strictly satisfy the energy constraints. Thus the Salter's condition for constraint qualification is satisfied, and strong duality holds between the primal and dual problems.

Therefore, we can solve the primal problem through the Lagrangian dual as

$$
\text { (LD) } \max W(\lambda, \rho, \mu)
$$

Through mathematical transformation, the Lagrangian dual function can be written as

$$
W(\lambda, \rho, \mu)=-\max \Phi(\mu, \rho)-\max \Psi(\lambda, \rho)
$$

where,

$$
\Phi(\mu, \rho)=\sum_{i \in \mathcal{N}} q_{i}\left(\sum_{j \in N(i)}\left(\mu_{j i}-\mu_{i j}\right)+\rho_{i} E_{i}-q_{i}\right)
$$

$$
\begin{aligned}
\Psi(\lambda, \rho)= & \sum_{s, d} \sum_{i \in \mathcal{N}} \sum_{(i, J) \in \mathcal{L}} \sum_{j \in J} g_{i J j}^{c s d}\left(\lambda_{j}^{c s d}-\lambda_{i}^{c s d}\right) \\
& \sum_{c \in C} \sum_{i \in \mathcal{N}} \sum_{(i, J) \in \mathcal{L}} f_{i J}^{c}\left[-\left(\rho_{i} e_{i}^{t}+\sum_{j \in J} \rho_{j} e_{j}^{r}\right)\right]
\end{aligned}
$$

Then the dual function can be decomposed into two subproblems

$$
\begin{gathered}
\max \Phi(\mu, \rho) \quad \text { s.t. Equation (13) } \\
\max \Psi(\lambda, \rho) \quad \text { s.t. Equations }(6)(7)(8)
\end{gathered}
$$

where $\Phi(\mu, \rho)$ is related to the lifetime, and named as lifetime optimization subproblem; $\Psi(\lambda, \rho)$ is determined by the flow distribution, and named as network optimization subproblem.

\section{Subgradient Algorithm}

Since the objective function of the primal problem (P3) is not strictly convex in the variable space, the Lagrangian dual function $L(q, g ; \lambda, \rho, \mu)$ is non-differentiable. Cutting plane and dual subgradient methods are two promising ways to solve non-differential optimization problems. However, the cutting plane method usually needs information of all dual solutions and so a central coordinator. Thus we employ the subgradient method to solve the dual problem, which often facilitates a distributed algorithm.

Suppose primal variables $q_{i}^{(k)}, g_{i J}^{c s d(k)}$ are obtained at the point $\lambda^{(k)}, \rho^{(k)}, \mu^{(k)}$, i.e.,

$$
\begin{gathered}
q_{i}^{(k)}=\arg \max _{E q n .(13)} \Phi\left(\mu^{(k)}, \rho^{(k)}\right) \\
g_{i J j}^{c s d(k)}=\arg \max _{E q n s .(6)(7)(8)} \Psi\left(\lambda^{(k)}, \rho^{(k)}\right)
\end{gathered}
$$

Given $q_{i}^{(k)}, g_{i J}^{c s d(k)}$, the subgradients of the dual problem (LD) on variables $\lambda_{i}^{c s d(k)}, \rho_{i}^{(k)}, \mu_{i j}^{(k)}$ are

$$
\begin{gathered}
h\left(\lambda_{i}^{c s d}\right)^{(k)}=\sum_{(i, J) \in \mathcal{L}} \sum_{j \in J} g_{i J j}^{c s d(k)}-\sum_{j \in \mathcal{N}} \sum_{(j, I) \in \mathcal{L}, i \in I} g_{j I i}^{c s d(k)}-\sigma_{i}^{c s} \\
h\left(\rho_{i}\right)^{(k)}=\sum_{c \in \mathcal{C}} \sum_{(i, J) \in \mathcal{L}} e_{i}^{t} f_{i J}^{c}+\sum_{c \in \mathcal{C}} \sum_{(j, I) \in \mathcal{L}, i \in I} e_{i}^{r} f_{j I}^{c}-q_{i} E_{i} \\
h\left(\mu_{i j}\right)^{(k)}=q_{i}-q_{j}
\end{gathered}
$$

The update of dual variables are as follows

$$
\begin{gathered}
\lambda_{i}^{c s d(k+1)}=\lambda_{i}^{c s d(k)}+\alpha_{k} h\left(\lambda_{i}^{c s d}\right)^{(k)} \\
\rho_{i}^{(k+1)}=\rho_{i}^{(k)}+\alpha_{k} h\left(\rho_{i}\right)^{(k)} \\
\mu_{i j}^{(k+1)}=\mu_{i j}^{(k)}+\alpha_{k} h\left(\mu_{i j}\right)^{(k)}
\end{gathered}
$$

where $\alpha_{k}>0$ is the step size.

According to [19], subgradient method is guaranteed to converge to the optimum of the convex problem if $\alpha_{k}$ satisfies

$$
\alpha_{k} \rightarrow 0, \quad \sum_{k=1}^{\infty} \alpha_{k}=\infty
$$




\section{Primary Recovery}

Notice that the dual function is non-differentiable, hence in general the optimal solution of the dual problem with subgradient method is not feasible to the primal problem. To overcome this problem, we use the convex combination method [20], which convexly combines dual solutions in previous iterations to guarantee the primal feasibility.

At each iteration $k$, we can compose the primal variable $x^{(k)}$ with

$$
x^{(k)}=\sum_{i=1}^{k} \beta_{i}^{(k)} x^{(i)}
$$

where $\beta_{i}^{(k)}$ is the convex combination weight satisfying $\sum_{i=1}^{k} \beta_{i}^{(k)}=1$, and $\beta_{i}^{(k)}>0, \forall i$. Note that $x^{(k)}$ is a general expression of $g_{i J}^{c s d(k)}$ and $q_{i}^{(k)}$.

We choose the convex combination weight as explained in Corollary 2 in [20].

$$
\beta_{i}^{(k)}=\frac{\alpha_{i}}{\sum_{j=1}^{k} \alpha_{j}}
$$

According to [20], the accumulation point $x_{i}^{(k)}$ generated via equation (31) is feasible to the primary problem (P3).

\section{E. Dual Function Analysis}

We first consider the subproblem in equation (20). Since $q_{i}$ can be limited by $[0 Q]$, the optimal solution of the quadric function can be easily obtained corresponding to different values of $x$.

$$
q_{i}=\left\{\begin{array}{cl}
0, & \text { if } x<0 \\
Q, & \text { if } x>Q \\
x / 2, & \text { otherwise. }
\end{array}\right.
$$

Since there exits the physical rate constraint in equation (6), the maximum value of the following linear equation (34) is always attained at the maximum positive point.

$$
\sum_{s \in \mathcal{S}^{c}} \sum_{j \in J} g_{i J j}^{c s d}\left(\lambda_{j}^{c s d}-\lambda_{i}^{c s d}\right)
$$

The corresponding effective flow rate $g_{i J j}^{c s d}$ is

$$
g_{i J j}^{c s d}=\left\{\begin{array}{c}
f_{i J}^{c}, \quad \text { if } s=s^{*}, j=j^{*}, \lambda_{j^{*}}^{c s^{*} d}-\lambda_{i}^{c s^{*} d}>0, \\
0, \quad \text { otherwise. }
\end{array}\right.
$$

where $s^{*}$ and $j^{*}$ are the source node and one of the nodes in $J$, which yield the maximum of $\lambda_{i}^{c s d}-\lambda_{j}^{c s d}$.

Thus, $\max \Psi_{1}$ is equivalent to

$$
\max \Psi_{3}=\sum_{i \in \mathcal{N}} \sum_{(i, J) \in \mathcal{L}} \sum_{c \in \mathcal{C}} f_{i J}^{c} \sum_{d \in \mathcal{D}^{c}}\left[\lambda_{j^{*}}^{c s^{*} d}-\lambda_{i}^{c s^{*} d}\right]^{+}
$$

where $[x]^{+}=\max (0, x)$.

The subproblem in equation (21), therefore, becomes the following form

$$
\max \Psi=\Psi_{3}+\Psi_{2}=\sum_{c \in C} \sum_{i \in \mathcal{N}} \sum_{(i, J) \in \mathcal{L}} f_{i J}^{c}(y+z)
$$

where,

$$
y=-\left(\rho_{i} e_{i}^{t}+\sum_{j \in J} \rho_{j} e_{j}^{r}\right) \quad z=\sum_{d \in \mathcal{D}^{c}}\left(\lambda_{j^{*}}^{c s^{*} d}-\lambda_{i}^{c s^{*} d}\right)^{+}
$$

Considering the constraint $\sum_{c \in C} f_{i J}^{c} \leq r_{i J}$ in equation (7), the optimal value of equation (37) can be written in the following manner

$$
\sum_{i \in \mathcal{N}} \sum_{(i, J) \in \mathcal{L}} r_{i J}\left[y\left(c^{*}\right)+z\left(c^{*}\right)\right]^{+}
$$

where $c^{*}$ is the commodity that maximize $y+z$. The corresponding physical commodity rate $f_{i J}^{c}$ is

$$
f_{i J}^{c}=\left\{\begin{array}{c}
r_{i J}, \quad \text { if } c=c^{*}, y\left(c^{*}\right)+z\left(c^{*}\right)>0 \\
0, \quad \text { otherwise. }
\end{array}\right.
$$

From the above derivations, the problem in equation (21) can be written as

$$
\max \sum_{i \in \mathcal{N}} \sum_{(i, J) \in \mathcal{L}} r_{i J}\left[y\left(c^{*}\right)+z\left(c^{*}\right)\right]^{+}
$$

which is a weighted link scheduling problem, and can be solved according to the specific interference model.

Using the primary interference model, the problem in equation (41) is equivalent to maximum weighted hypergraph matching, which can be solved with an approximated algorithm in a distributed manner as proposed in [10]. The complexity of solving the problem depends on variable spaces that determine the achievable rate region $\operatorname{Co}(\Pi)$.

According to above analysis, we can give the distributed algorithm based on subgradient method as in Algorithm 1.

Algorithm 1 Subgradient Method to Solve the Lifetime Maximization Problem with Inter-Session Network Coding

STEP 0: Initialization. Start with a initial point $\lambda, \rho, \mu$. Choose an sequence of positive step size $\alpha_{k}$ satisfying requirements in Equation (30). Set $k=1$.

STEP 1: Dual Variables Transfer. For each node $i$, the Lagrangian variables are broadcasted to its neighboring nodes.

STEP 2: Primal Variables Computation and Transfer. Compute the primal variables according to equations (22)(23), convexly combine the current primal solution with previous ones through $\beta_{i}$ in Equation (32), and transfer the final results to neighboring nodes.

STEP 3: Subgradient Calculation. Compute subgradients according to equations (24)(25)(26). If all subgradients are equal to 0 , then the optimization problem is solved, and STOP.

STEP 4: Dual Variables Update. Calculate the dual variables according to equations (27)(28)(29), and go to STEP 1. 


\section{Evaluation}

For space limitation, we evaluate the distributed network coding algorithm for lifetime maximization on the network topology as shown in Figure 1. There are two flows: $\{1\} \rightarrow$ $\{5,7\},\{2\} \rightarrow\{5,7\}$, which constitute a commodity. The source rates of both source nodes are 0.2 , while the transmission rate of each multicast is set to be 1. It is obvious that the source rates can be supported by the network.

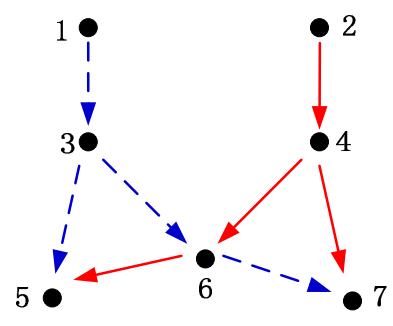

Fig. 1. Flows and commodities.

The initial energy of each node is assumed to be 100 joule. Considering different energy costs of transmitting and receiving, the transmission energy cost $e_{i J}^{t}$ of node $i$ is assumed to be 2 joule, while the receiving energy $\operatorname{cost} e_{j I}^{r}$ is assumed to be 1 joule for each node $i$. No power control is considered in following simulation.

The initial values of $\lambda_{i}^{c s d}, \rho_{i}$, and $\mu_{i j}$ are assumed to be 0 . The step sizes $\alpha_{k}$ of iterations on $\lambda_{i}, \rho_{i}$, and $\mu_{i j}$ are set to $\max \left(10^{-4}, 10^{-3} / \sqrt{k}\right)$, where $k$ is the iteration index.

Variation of $q_{6}$ with iterations is shown in Figure 2. $q_{i}$ of other nodes with $i \neq 6$ is similar and omitted here. As shown in the figure, the values of $q$ converge to the expected ones, after a thousand iterations. Since the topology is very simple, we can get the energy cost easily. Without network coding, the bottleneck node 6 has to transmit and receive twice, then $q=(0.2 * 2 * 2+0.2 * 1 * 2) / 100=0.012$. With linear network coding, node 6 has to receive twice and transmit once, which results in $q=(0.2 * 2 * 1+0.2 * 1 * 2) / 100=0.008$. The convergence in Figure 2 matches our expectation very well. And the lifetime with inter-session network coding is about $50 \%$ larger than that without network coding.

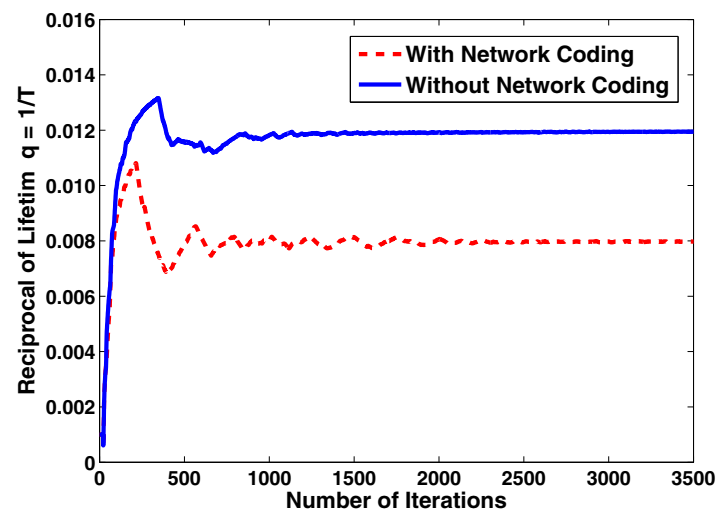

Fig. 2. Convergence of lifetime reciprocal $q$.

\section{Conclusion}

In this paper, we have investigated lifetime maximization for energy constrained networks with inter-session network coding. We formulate the lifetime maximization as a linear programming problem and solve it with the dual subgradient method in a distributed manner. The algorithm is evaluated on a specific topology. Results of simulation well match that of the optimization analysis, and lifetime is extended about $50 \%$ with inter-session network coding.

\section{REFERENCES}

[1] J.-Hoo, Chang, and L. Tassiulas, "Maximum lifetime routing in wireless sensor networks," IEEE/ACM Trans. Networking, vol. 12, no. 4, pp. 609619, Aug. 2004.

[2] R. Madan, and S. Lall, "Distributed algorithms for maximum lifetime routing in wireless sensor networks," IEEE Trans. on Wireless Communications, vol. 5, no. 8, pp. 2185-2193, Aug. 2006.

[3] R. Ahlswede, N. Cai, S.-Y. R. Li, and R. W. Yeung, "Network information flow," IEEE Trans. on Information Theory, vol. 46, no. 4, pp. 1204-1216, Jul. 2000.

[4] S-Y. R. Li, R. W. Yeung, and N. Cai, "Linear network coding," IEEE Trans. on Information Theory, vol. 49, no. 2, pp. 371-381, Feb. 2003.

[5] T. Ho, M. Medard, and R. Koetter, "A random linear network coding approach to multicast," IEEE Trans. on Information Theory, vol. 52, no. 10 , Oct. 2006.

[6] T. Ho, and H. Viswanathan, "Dynamic algorithms for multicast with intra-session network coding," IEEE Trans. on Information Theory, vol. 55, no. 4, pp. 797-815, Feb 2009.

[7] S. Katti, H. Rahul, W. Hu, D. Katabi, M. Medard, and J. Crowcroft, "XORs in the air: Practical wireless network coding," IEEE/ACM Trans. on Networking, vol. 16, no. 3, pp. 497-510, Jun. 2008.

[8] D. Traskov, N. Ratnakar, D. S. Lun, etc., "Network coding for multiple unicasts: An approach based on linear optimization," in Proc. IEEE ISIT, Seattle, USA, Jul. 2006.

[9] L. You, P. Wu, Z. Pan, H. Hu, M. Song, and J. Song, "Cross-layer optimization of wireless multi-hop networks with network coding," in Proc. WCSP, Nanjing, China, Nov. 2009.

[10] T. Cui, L. Chen, and T. Ho, "Distributed optimization in wireless networks using broadcast advantage," in 46th IEEE Conference on Decision and Control, New Orleans, LA, USA, Dec 2007.

[11] Nagajothy. M, and D. Radha, "Network lifetime enhancement in wireless sensor network using network coding," in International Conference on Control, Automation, Communication and Energy Conservation, 2009.

[12] Y. E. Sagduyu and A. Ephremides, "Joint scheduling and wireless network coding," in Proc. WINMEE, RAWNET and NETCOD, 2005. [Online]. Available: http://netcod.org/papers/01SagduyuE-final.pdf

[13] M. Nagajothy, and S. Radha, "Network lifetime Enhancement in Wireless Sensor Networks Using Network Coding," in Proc. IEEE INCACEC, Jun. 2009, pp. 1-4.

[14] A. Khreishah, C.-C. Wang, and N. B. Shroff, "Cross-layer optimization for wireless multihop networks with pairwise intersession network coding," IEEE Journals on Selected Areas in Communications, vol. 27, pp. 606-621, Jun. 2009.

[15] B Hajek, and G. Sasaki, "Link scheduling in polynomial time," IEEE Trans. on Information Theory, vol. 34, no. 5, pp. 810-917, 1988.

[16] C. Joo, X. Lin, and N. B. Shroff, "understanding the capacity region of the greedy maximal scheduling algorithm in multi-hop wireless networks," in IEEE Proc. INFOCOM, 2009.

[17] J.-H. Chang, and L. Tassiulas, "Energy conserving routing in wireless ad-hoc networks," in Proc. IEEE INFOCOM, 2000.

[18] L. Zhang, S. Chen, Y. Jian, and Y. Fang, "Distributed progressive algorithm for maximizing lifetime vector in wireless sensor networks," in IEEE Proc. INFOCOM, 2009.

[19] S. Boyd, L. Vandenberghe, Covex Optimization. Cambridge University Press, 2004

[20] H. D. Sherali, and G. Choi, "Recovery of primal solutions when using subgradient methods to solve lagrangian duals of linear programs," Operations Research Letters, vol. 19, pp. 105-113, 1996. 